\title{
Optimizing Parameters of Rail-induced Vibration Isolation Trench Using Simplified FEM Approach
}

\author{
Liang Wang, Yu-Bin Wu, Rui-Xiang Song and Ya-Nan Wu \\ State Environmental Protection Engineering Centre for City Noise and Vibration Control, Beijing Municipal Institute of Labour \\ Protection, Beijing 100054, China
}

\begin{abstract}
A simplified approach is proposed to reduce computational cost in conventional parametric optimization of open or in-filled trenches isolating rail-induced structural vibrations. In particular, it stands on an FEM-based hybrid optimization scheme consisting of multiple two-dimensional models and one global three-dimensional model. First, representative planar FE (finite element) models orthogonal to the rail-direction are identified. For each section, the sensitivity of the trench's design parameters, such as geometry and backfill materials, to its vibration screening effect is respectively evaluated. Second, a full trench along the rail-direction is determined according to the two-dimensional optimization result. The global performance of the optimal trench is simulated in the three-dimensional model and finally becomes a reference for practical design. By optimizing the design parameters of a case study project, the proposed approach has shown the capability of solving complex engineering problems at a minimum computational cost, therefore is applicable in determining design parameters of rail-induced vibration isolation trenches.
\end{abstract}

Key words: Rail-induced environmental vibration, vibration isolation trench, rail-property development, numerical simulation, engineering design optimization.

\section{Introduction}

Recently, due to scarcity of the developable land, an increasing number of commercial and residential properties have been built adjacent to rail transit lines in metropolises. While it provides transportation convenience, the environmental vibration resulting from train tracks may generate annoyance to nearby dwellers.

Vibration isolation trench with open or in-filled material is a kind of wave barrier set between the vibratory source and receivers. If designed with appropriate parameters, the trench is able to reflect and disperse the energy propagated from the vibratory source, and therefore reduce groundborne vibration transmitted to a nearfield protection subject. As exampled in Fig. 1, the trench may locate next to the source, the receiver, or somewhere in between. When adjacent to the source it serves as an active screen

Corresponding author: Liang Wang, assistant researcher, Ph.D., research fields: rail-induced vibration and control, and structural health monitoring. shielding vibration from the track and a shallow buried depth is often necessary; when close to the receiver, conversely, the target building is passively isolated and the trench may need to be buried deep enough to fully screen transmitted and diffracted waves [1].

So far, a great deal of theoretical and experimental work has been done to study the passing train-induced vibration reduction effectiveness of the barrier, and many have discussed the influence of varying design parameters. Beskos et al. [2] used frequency-domain two-dimensional BEM (boundary element models) and studied the effectiveness of open and in-filled trenches isolating ground borne vibration. Zoccali et al. [3] compared the vibration reduction capacity of the in-filled anti-vibration trench with varying input parameters such as length and material types. Garinei et al. [4] conducted in-situ and prototype experiments and the efficiency of isolating trenches where various types and shapes were measured.

In the literature, FE (finite element) modeling is a popular tool to solve structural optimization problems. 


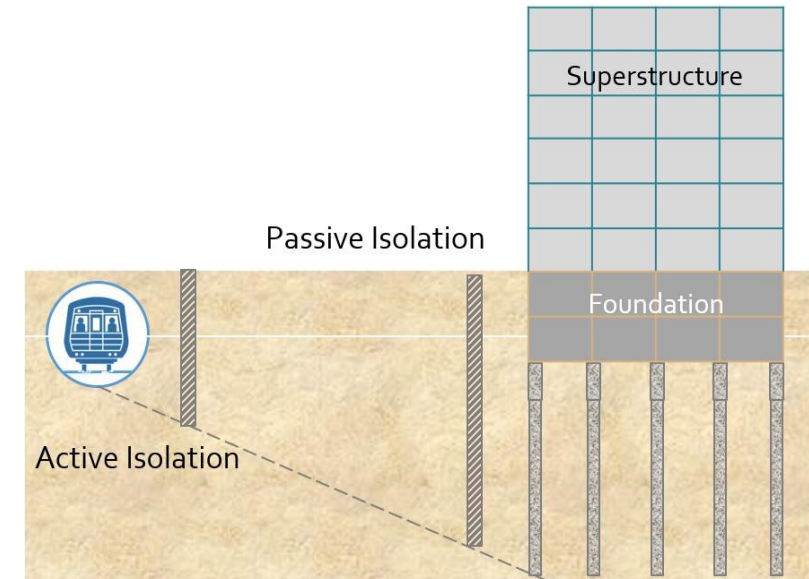

Fig. 1 Rail-induced vibration isolation trenches.

With an FE model it is convenient to evaluate multiple values of a parameter in a desired range and find the optimal value, and the sensitivity of particular parameters against the trench's isolation performance has been widely investigated using two-dimensional models. In the situation that a protection subject cannot be simplified in a single two-dimensional plane, however, one has to reach a full 3D model which features the vibratory source, the medium (most likely soil) and the receivers. Consequently, the optimization may be very difficult to proceed due to a high computational cost.

In order to overcome the efficiency problem, this paper proposes a simplified optimization scheme aiming to facilitate the process by using several plane-strain FE models along with the spatial FE model. The proposed optimization scheme is later illustrated by an in-situ case study problem.

\section{Optimization Parameters}

\subsection{Dimensional Configuration}

For all kinds of the trench, parameters required to be optimized are mainly dimensional configuration along with in-filled material characteristics. Previous study has concluded that within geometric factors distance from the source and the buried depth is mostly sensitive to the isolation effect $[5,6]$. Generally, the propagated vibration is likely to be screened by a trench close to either the receiver or the source. Bo et al. [6] report that better performance is achieved from a deeper trench but the improvement becomes insignificant beyond a certain depth, and Alzawi [7] concluded that the influence of width change is limited except in the condition of shallow trenches.

\subsection{In-filled Material}

Material properties are dominant parameters directly affecting the isolation performance of a trench. According to the elastic wave theory [8], how a wave transmits through an interface is determined by the WIR (wave impedance ratio) between the two-side materials of the interface. Theoretically, the trench with extremely large or small WIR against the transmission medium is likely to have the best isolation performance. If the ratio equals to one, inversely, the trench may have no effect at all. Wang [9] investigated the in-filled of gravel, concrete, flyash, geofoam, and rubber and concluded that concrete gives the best performance as the stiff materials while geofoam stands out among the flexible materials. Further compare shows that geofoam outweighs concrete as the most appropriate in-filled material. Commonly used geofoam materials as the vibration isolation trench are EPS (expanded polystyrene) [6,7] and polyurethane [10].

\section{Simplified FEM-Based Optimization}

\subsection{Hypotheses}

To begin, the following hypotheses are made on the basis of characteristics in rail-induced structural vibration problems:

(1) In the direction perpendicular to the rail track there exist a set of planar models where the out-of-plane mass and stiffness is constant for a limited length, and the wave transmitting in each such plane is independent;

(2) The moving train load is decomposable as the combination of a series of sinusoidal waves. The sinusoidal loads have consistent spectral features with the actual moving loads;

(3) Different medium interfaces are assumed to be fully bonded in the FEM. 


\subsection{Simplified Optimization Scheme}

The simplified optimization is mainly executed on a series of the updated planar models with high computational efficiency. First, construct a full spatial model consisting of the vibratory source, the transmission medium, and the receiver(s). The full model is expected to be modified by field test data and essentially reveal the actual propagation of the vibration wave energy. Second, pick out representative planes normal to the track direction and prepare plane-strain models respectively. Third, update the planar models with the response of particular checkpoints in the $3 \mathrm{D}$ model under the actual moving loads. The sinusoidal loads are then applied on the refined planar models that are ready for optimization computations. Next, determine candidate parameters with relevant boundaries. Finally carry out the optimization with varying parameters and filter out the optimal parameters of the isolation trench. Fig. 2 is the working flowchart of the proposed optimization scheme.

Because the optimization in 2D models is to seek the trench's appropriate parameters leading to the best screening effect, the calculated insertion loss in vibration energy is relative and can hardly represent a continuous trench's performance in the field. Thus, in order to evaluate the trench's global effectiveness a comprehensive analysis in the spatial FEM may be called for when the optimization process completes for all plane-strain models.

\section{Case-Study Project}

The proposed analytical steps are illustrated by an in-situ case study project shown in Fig. 3, where two high-rise residential buildings (B-10\# and B-11\#) are subject to vibration from the subway lines to the north. The 27-storey frame shear wall building has 3-floor under-ground, and the elevation of the bottom of the foundation is $-10.6 \mathrm{~m}$. The longitudinal stiffness of the structure is distributed in parallel with the direction of the track to the north with a gap distance of about $30 \mathrm{~m}$, where the track is inclined from $-4.0 \mathrm{~m}$ to the surface.

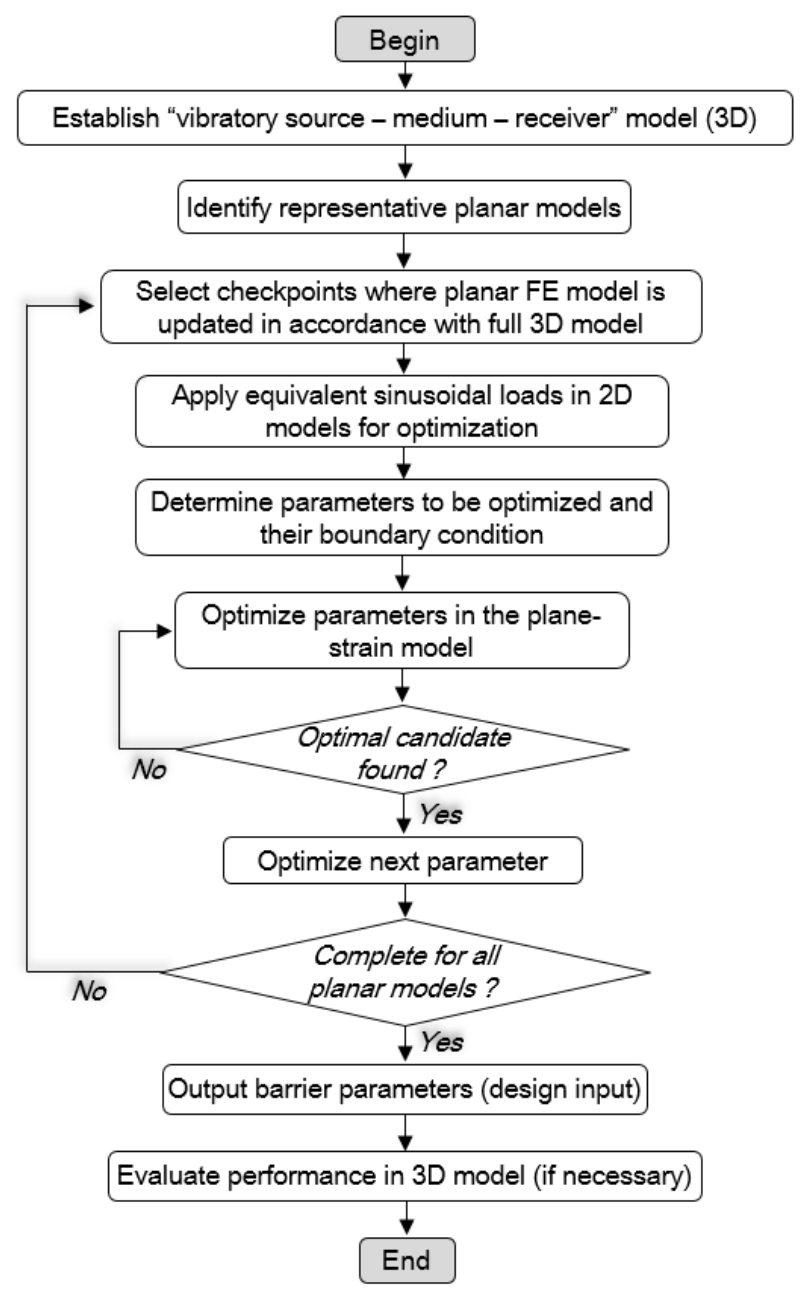

Fig. 2 Scheme of the proposed optimization method.

To reduce the vibration transmitting to the building, a trench is planned between the railway and the structures. Available land that can be utilized for constructing a continuous trench has been identified in Fig. 3a, and Fig. 3b shows the profile of the vibratory source, soil, and the receivers. The borehole log of the site is shown in Fig. 4. The passing speed of the train is $20 \mathrm{~km} / \mathrm{h}$.

\subsection{Numerical Modelling and Updating}

A full ANSYS spatial model of the rail tracks, tunnel, soil medium, and the protection building is simulated. Several orthogonal plane-strain models representing constant stiffness of a limited range along the track are identified. Fig. 5 shows one of such sections in two-dimensional FEM with two updating checkpoints. 
The train loads are applied according to Eq. (1).

$$
P=\sum_{k=1}^{N} P\left(\omega_{k}\right), \quad P\left(\omega_{k}\right)=P_{0}^{k} \cdot e^{i \omega_{k} t}
$$

$P_{0}$ and $\omega$ are magnitude of loads in Newton and circular frequency in $\mathrm{rad} / \mathrm{s}$, respectively. $N$ is the number of frequency resolution lines. For the sake of computational efficiency and minimum accuracy sacrifice, the buildings to the south of B-10\# and B-11\# are simplified as mass blocks in the plane-strain model. To ensure the simplified model be consistent with its counterpart spatial model, the acceleration responses in spectrum at the check-points are utilized as the criterion in the model updating. Firstly, spatial train loads are decomposed in frequency domain, and the spectral loads then become an initial value for the sinusoidal function in Eq. (1), by which the responses at the checkpoints are calculated and compared. Since the rail-induced vibration energy is relatively small, the entire model can be assumed as a LTI (linear time invariant) system. In the case, a discrepancy is found for $k^{\text {th }}$ frequency, $P_{0}{ }^{k}$ will iterate for the next value and so on. Fig. 6 shows the comparison results of the case study model after updating at the checkpoints.

\subsection{Optimization and Results}

For the case study, several sensitive design parameters such as the trench's horizontal distance to the source (L), buried depth (D), and in-filled material properties (open or in-filled). As the trench width is found not crucial to the isolation performance, a constant width of $1 \mathrm{~m}$ is adopted in all analysis. To make the analysis independent against the frequency of the exciting force, geometry properties are normalized to the Rayleigh wavelength of the field soil. Since the Rayleigh wave speed is $225 \mathrm{~m} / \mathrm{s}$, and a prior evaluation shows that the structures will receive the most vibration energy at $33 \mathrm{~Hz}$, the wavelength can be calculated as $\lambda_{R}$ $=V_{\mathrm{r}} / f=225 \mathrm{~m} / \mathrm{s} / 33 \mathrm{~Hz}=6.8 \mathrm{~m}$. Dimensionless optimization candidates are listed in Table 1.

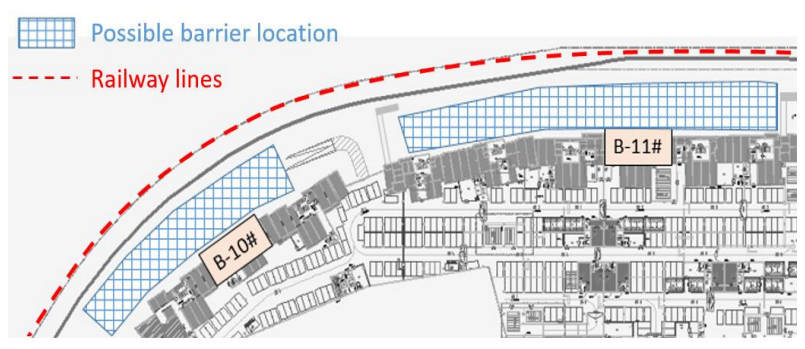

(a) Project layout

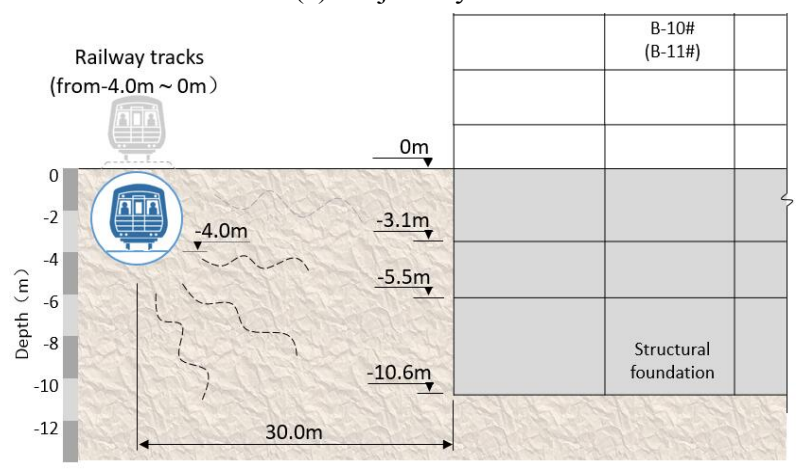

(b) Vertical cross-section

Fig. 1 Rail-induced vibratory source and receivers.

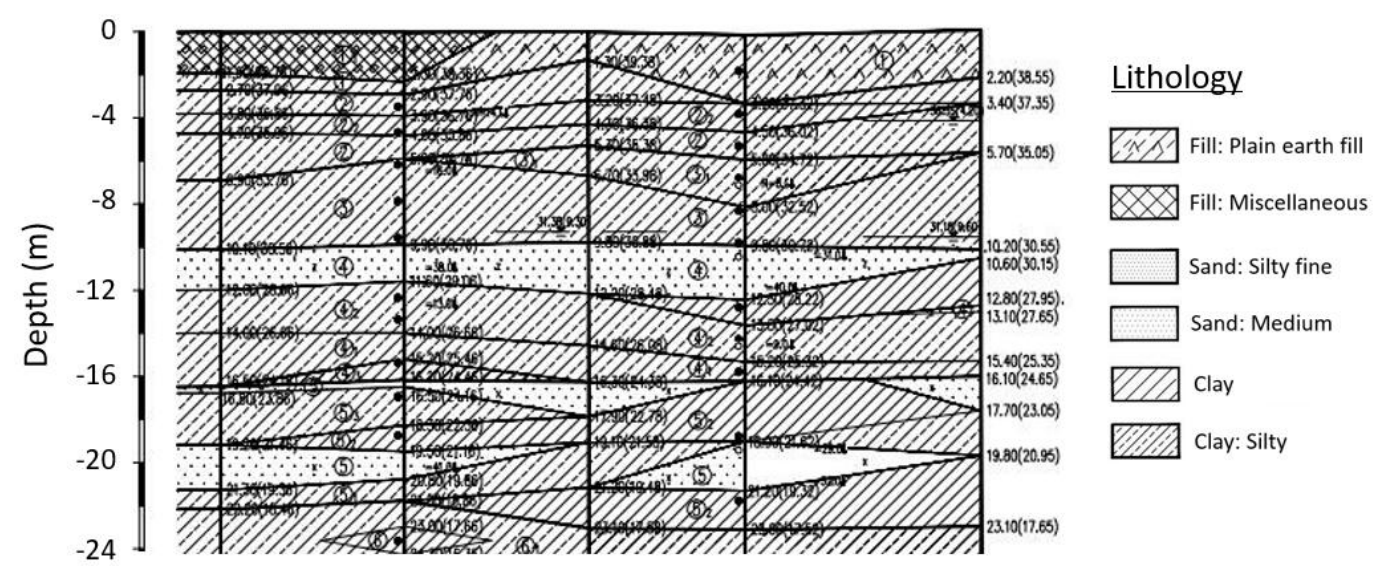

Fig. 2 Boring log of the site near the receivers. 


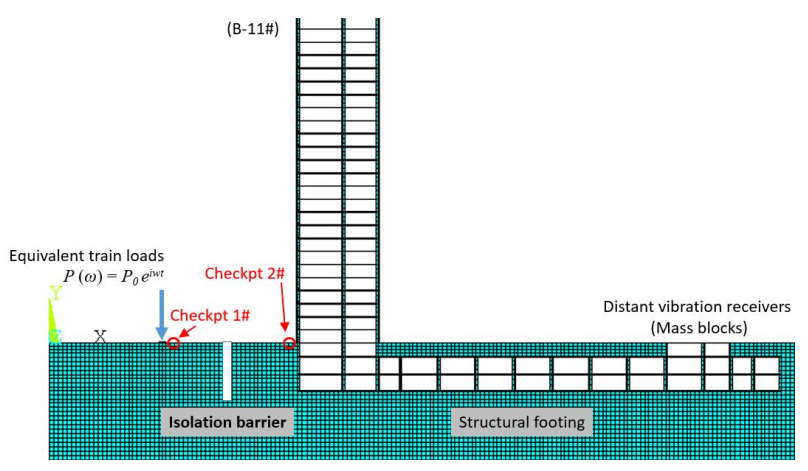

(a) Checkpoints in the 2D FE model

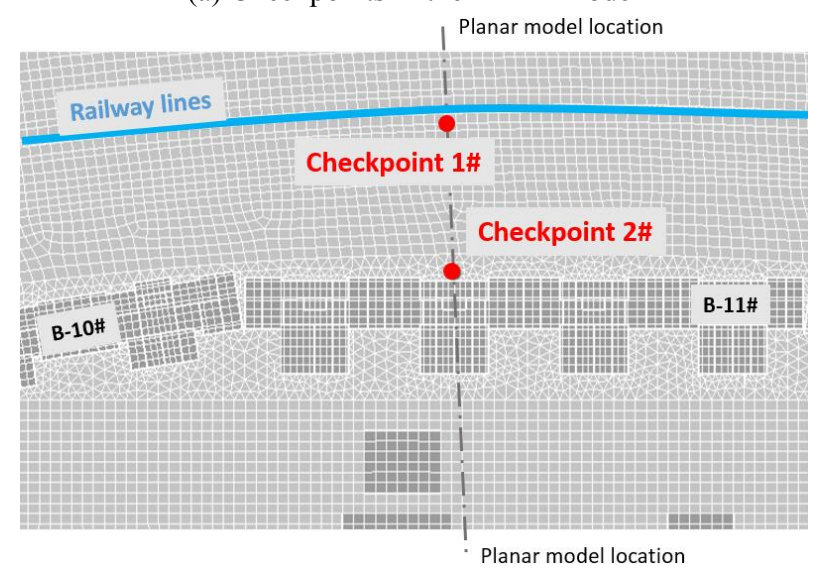

(b) Corresponding checkpoints in the 3D FE model

Fig. 5 Checkpoint location of the simplified model.

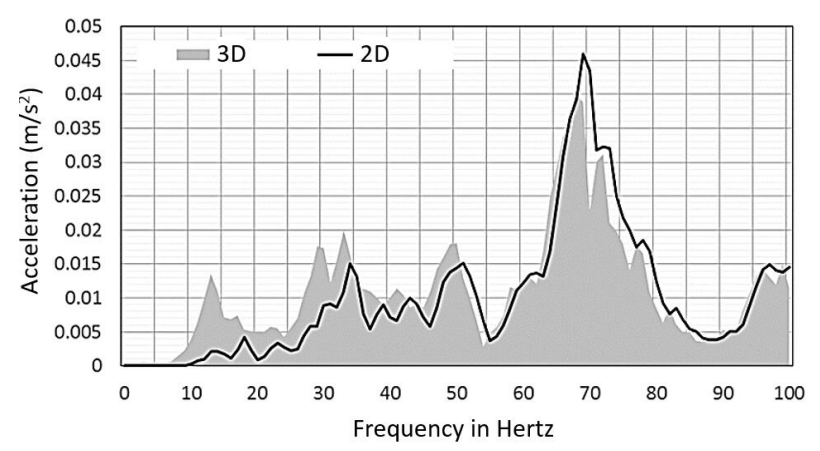

(a) Checkpoint $1 \#$ result

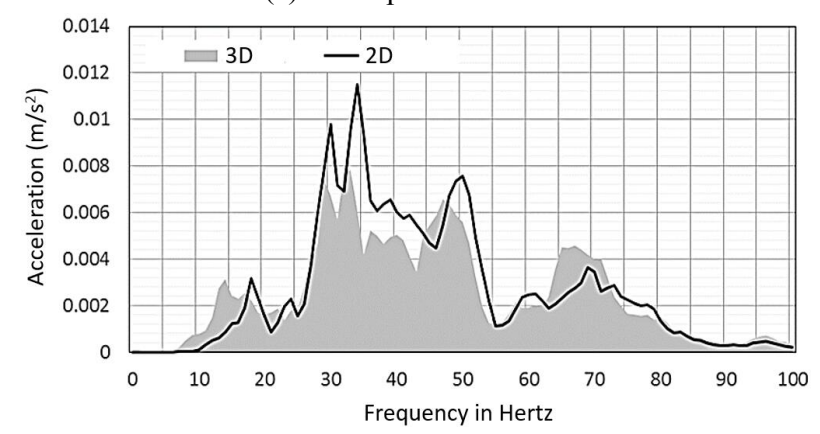

(b) Checkpoint $2 \#$ result

Fig. 6 Spectral response comparison after model updating.
Table 1 Parametric optimization candidates (normalized to Rayleigh wavelength).

\begin{tabular}{ll}
\hline Parameters & Optimization candidates \\
\hline$L^{\prime}=L / \lambda_{R}$ & $0.74,1.47,2.21,2.94,3.68$, and 4.26 \\
$D^{\prime}=D / \lambda_{R}$ & $0.44,1.59^{*}$, and 2.21 \\
$W^{\prime}=W / \lambda_{R}$ & 0.15 \\
Material & EPS-12, -15, -19, -22, -29, -39, -46, and \\
\hline
\end{tabular}

*identical to the elevation at the bottom of the structure's foundation.

Implementing the proposed optimization scheme, vertical acceleration at the center of the structure's floors is employed to judge optimal values for each parameter whose varying values are stated in Table 1. The judgement index is given by Eq. (3).

$$
\bar{S}_{Z}=\frac{1}{N_{1}} \sum_{L=1}^{N_{1}} \frac{V L_{z \max }}{V L_{z \max }^{0}}
$$

$V L_{Z \max }$ and $V L_{Z \max }^{0}$ denote the vertical vibration acceleration $(\mathrm{dB})$ with and without a trench. In the optimization, it takes only about one hour for the 2D model to finish a session of calculation while with the same hardware and software require $51 \mathrm{~h}$ to finish the task on the 3D model. In other words, with the same computational cost the simplified scheme is able to include more optimization candidates.

Table 2 lists the performance comparison of anti-vibration trench adopting different parameters, where the performance is evaluated by an averaged judgement index of all floors. In theory, void (open) trenches tend to possess most effectiveness than the in-filled, which is generally agreed with the result of the case study. Moreover, the result of candidate 1-12 in Table 2 implies that the deep void trench $(10.8 \mathrm{~m}$ or $15 \mathrm{~m}$ ) retains better isolation than the shallow trench (3 $\mathrm{m})$. Additionally, the best performance is sought with 5 $\mathrm{m}$ horizontal distance to the source, where the vibration is reduced $14.3 \%$. Considering the site limitation of such a close distance as $5 \mathrm{~m}$, a secondary optimal value of $20 \mathrm{~m}$, where $11.3 \%$ vibration is isolated, can be chosen as the optimal open trench location,

From a structural point of view, the in-filled trench that has higher transverse stability than the open is 
Table 2 Comparative results of the trench with various design parameters.

\begin{tabular}{llllll}
\hline $\begin{array}{l}\text { Candidate } \\
\text { No. }\end{array}$ & Material & $L^{\prime}$ & $D^{\prime}$ & $W^{\prime}$ & $\bar{S}_{Z}$ \\
\hline 1 & Void & 0.74 & 2.21 & 0.15 & 0.857 \\
2 & Void & 1.47 & 2.21 & 0.15 & 0.903 \\
3 & Void & 2.21 & 2.21 & 0.15 & 0.858 \\
4 & Void & 2.94 & 2.21 & 0.15 & 0.887 \\
5 & Void & 3.68 & 2.21 & 0.15 & 0.916 \\
6 & Void & 4.26 & 2.21 & 0.15 & 0.883 \\
7 & Void & 0.74 & 1.59 & 0.15 & 0.878 \\
8 & Void & 1.47 & 1.59 & 0.15 & 0.942 \\
9 & Void & 2.21 & 1.59 & 0.15 & 0.911 \\
10 & Void & 2.94 & 1.59 & 0.15 & 0.899 \\
11 & Void & 3.68 & 1.59 & 0.15 & 0.923 \\
12 & Void & 4.26 & 1.59 & 0.15 & 0.925 \\
13 & Void & 2.21 & 0.44 & 0.15 & 0.951 \\
14 & Void & 2.94 & 0.44 & 0.15 & 0.964 \\
15 & EPS12 & 2.94 & 2.21 & 0.15 & 0.895 \\
16 & EPS15 & 2.94 & 2.21 & 0.15 & 0.901 \\
17 & EPS19 & 2.94 & 2.21 & 0.15 & 0.908 \\
18 & EPS22 & 2.94 & 2.21 & 0.15 & 0.912 \\
19 & EPS29 & 2.94 & 2.21 & 0.15 & 0.920 \\
20 & EPS39 & 2.94 & 2.21 & 0.15 & 0.926 \\
21 & EPS46 & 2.94 & 2.21 & 0.15 & 0.929 \\
\hline
\end{tabular}

more applicable to be constructed and maintained in practice. Thus, the trench with various in-filled EPS materials listed by ASTM [11] is sequentially evaluated. From the analysis candidate 15 of an EPS-12 material is found to wipe out $10.5 \%$ vibration. And this effectiveness is very close to the performance of its open trench counterpart.

\section{Conclusions}

This paper demonstrates a simplified parametric optimization scheme for the soil embedded trenches designed to reduce the vibration of structures transmitted from nearby railway tracks. The optimization has been implemented on simplified FE models orthogonal to the track. Comparing with the process conventionally undertaken on a spatial model, the proposed approach facilitates the optimization and evaluation of the trench's parameters with little computational cost. The proposed optimization process is illustrated through an in-situ case study.
With a quick convergence, the results show that a trench of $15 \mathrm{~m}$ depth, $1 \mathrm{~m}$ width, and $5 \mathrm{~m}$ distance to the source will have the best isolation performance. Future work will focus on simplification limitations of the approach.

\section{Acknowledgments}

This work is jointly supported by Beijing Academy of Science and Technology under the Special Supportive Grant for Returned Overseas Scholars No. OTP-2014-003 and Beijing Municipal Public Finance Project PXM2016_178304_000011.

\section{References}

[1] Lombaert, G., Degrande, G., François, S., and Thompson, D. J. 2015. "Ground-Borne Vibration due to Railway Traffic: A Review of Excitation Mechanisms, Prediction Methods and Mitigation Measures." Notes on Numerical Fluid Mechanics and Multidisciplinary Design 126: 253-87.

[2] Beskos, D. E., Dasgupta, B., and Vardoulakis, I. G. 1986. "Vibration Isolation Using Open or Filled Trenches Part I: 2-D Homogeneous Soil." Computational Mechanics 1: 43-63.

[3] Zoccali1, P., Cantisani, G., and Loprencipe, G. 2015. "Ground-Vibrations Induced by Trains: Filled Trenches Mitigation Capacity and Length Influence." Construction and Building Materials 74: 1-8.

[4] Garinei, A., Risitano, G., and Scappaticci, L. 2014. "Experimental Evaluation of the Efficiency of Trenches for the Mitigation of Train-Induced Vibrations." Transportation Research Part D: Transporta and Environment 32: 303-15.

[5] Real, J. I., Galisteoa, A., Real, T., and Zamorano, C. 2012. "Study of Wave Barriers Design for the Mitigation of Railway Ground Vibrations." Journal of Vibroengineering 14 (1): 408-22.

[6] Bo, Q., Ali, L., and Irini, D. M. 2014. "Numerical Study of Wave Barrier and Its Optimization Design." Finite Elements in Analysis and Design 84: 1-13.

[7] Alzawi, A. M. A. 2011. "Vibration Isolation Using In-Filled Geofoam Trench Barriers." Ph.D. thesis, The University of Western Ontario, Paper 265. http://ir.lib.uwo.ca/etd/265.

[8] Richart, F. E., John, R. H., Richard, D. W., and Prentice-hall Inc. 1970. Vibrations of Soils and Foundations. Monograph, Transport and Road Research Laboratory (TRRL).

[9] Wang, T. Y. 2008. "Study on Vibration Isolation and 
Vibration Isolation of Buildings Caused by Subway Operation.” Ph.D. thesis, Tongji University.

[10] Makiuchi, K., Minegishi, K., Mizutani, Y., and Mizukami, M. 2002. "Vibration Reduction Methods Using Ground Wave Propagation Cut Walls and Their Effects." In
Proceedings of the Japan National Conference on Geotechnical Engineering, 2405-6.

[11] ASTM. 2015. D6817/D6817M-15, Standard Specification for Rigid Cellular Polystyrene Geofoam, ASTM International, West Conshohocken, PA. 